\title{
Computer Tomography from Micro-Electronics to Assembled Products
}

\author{
Keith Bryant ${ }^{1, *}$, Ragnar Vaga ${ }^{2}$ \\ ${ }^{1}$ SMT Solutions. Johannesburg, South Africa \\ ${ }^{2}$ Yxlon International, Hamburg, Germany
}

\section{A R T I C LE IN F O \\ Article history: \\ Received: 20 April, 2017 \\ Accepted: 06 June, 2017 \\ Online: 30 June, 2017}

Keywords:

Computer Tomography

Micro-electronics

$X$-ray technology

\begin{abstract}
A B S T R A C T
Traditional CT in our industry has been limited to Business card sized samples, due to the Cone Beam x-ray systems used by Electronics manufacturing companies. Inclined or Partial CT provides a slightly different solution showing layers or slices in $2 D$ very well, but due to the partial nature of the scans does not produce very accurate $3 D$ reconstructions. This seminar will look at more sophisticated $x$-ray systems, including dual tube units, which can image at sub-micron level and have the ability to build an accurate and detailed 3D image of a tablet or smart phone without any stitching or joining of images. With high quality reconstruction software, these images can easily be manipulated to allow key features or failure sites to be easily seen. These systems are being used in Failure Analysis but also in NPI and in the design and development process as CAD data can be overlaid and metrology is also possible with some systems.
\end{abstract}

\section{Introduction}

\section{Traditional Electronics CT}

Within electronics manufacturing and failure analysis inspection 2D inspection is the primary method of $\mathrm{x}$-ray inspection and traditionally systems were only offering this. CT or $3 \mathrm{D}$ then became an option and quality varied dramatically dependant on many factors including: quality of image, stability of x-ray tube, reconstruction software, computing power etc. Time to make a 3D image was also long and to get detailed images could take many hours. This made it something which had very little use in the real world of manufacturing.

However due to the design of the systems and the technology used the biggest issue with CT was that the sample size was roughly the dimensions of a Business Card. This was due to the simple fact that to get good magnification the sample has to be close to the X-ray tube and to build the CT image the sample has to be rotated within the beam of the $\mathrm{x}$-ray tube.

Cone beam technology is standard in the machines used for electronics inspection.

${ }^{*}$ Corresponding Author: Keith Bryant, SMT Solutions. Johannesburg, South Africa |Email: keith.dage@gmail.com

www.astesj.com

https://dx.doi.org/10.25046/aj0203117

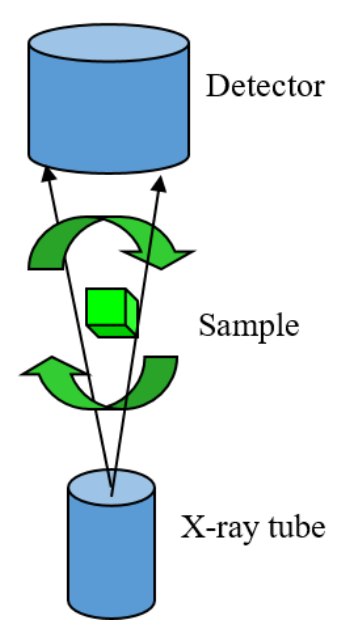

Figure 1 Conventional CT x-ray layout

The tube is at the bottom of the system, the sample held by some form of manipulator and a collector at the top of the system. Or the other way around in some systems, however this can cause issues with the sample colliding with the tube. So, in this type of systems CT is normally a failure analysis technique, unless you are working at the component level. 


\section{Inclined or partial CT}

This is a quite recent technology, the main benefit is that it works by focussing on an area of interest on an assembly or product, the only size limit is the inspection area of the system. The area of interest is set within the $\mathrm{x}$-ray cone beam and the sample or the detector is rotated.

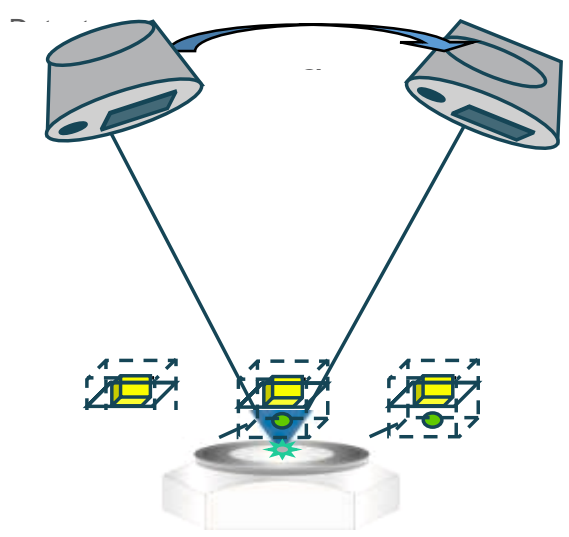

Figure 2 ICT manipulation

A larger number of images are taken, for instance one every degree, these are then processed to make a number of slices. This allows the operator to drill down through the number of slices to check interfaces and other key areas within the images. It is also used for separating components on the top and bottom sides of the board where complex components are 'mirrored' causing issues for traditional 2D x-ray systems. This is due to overlapping images of the components even at an angled view which can cause confusion or make voiding calculations impossible.

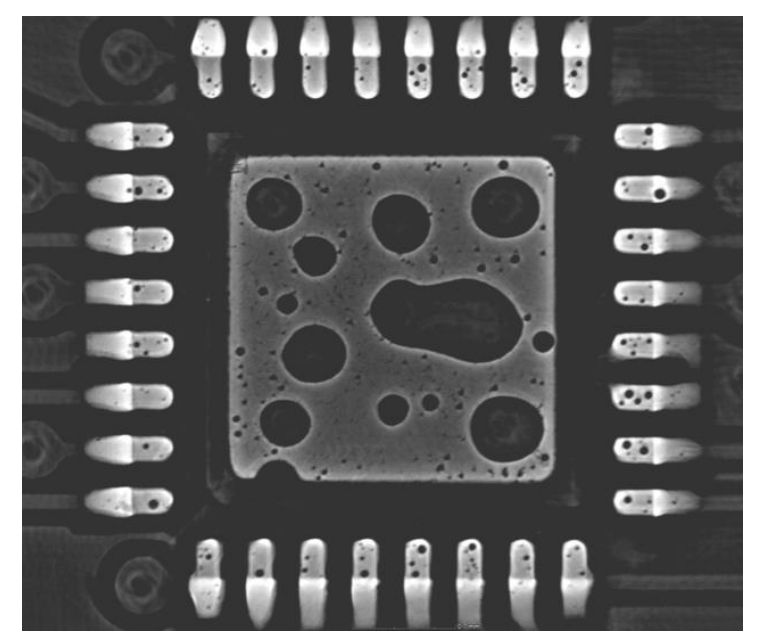

Figure 3 ICT slice showing QFN interface

However, as the captured images are taken at an angle and not by rotating the sample 360 degrees within the beam some data is missing. This means that when the $3 \mathrm{D}$ reconstruction is done it is not accurate in some areas as the data is incomplete. To overcome this some systems guestimate what should be there, which makes the image look more real but may not be accurate.

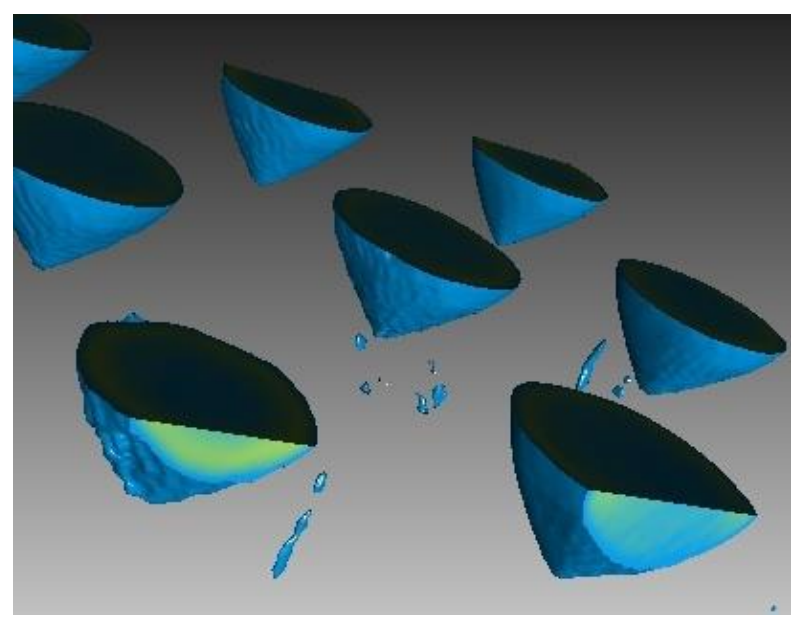

Figure 4 BGA balls showing lack of data

The figure above shows a section through some BGA balls, where the cross section is taken the $3 \mathrm{D}$ reconstructed image is accurate but the pointed area of the balls is inaccurate and is a result of lack of gathered data in this area.

\section{Cross Over Systems}

\section{Background}

The lines between NDT and Electronics x-ray inspection have become blurred as smaller features need to be seen in NDT and assembled product $\mathrm{CT}$ is being requested by more and more electronic manufacturing companies.

This has led to more flexible platforms being developed which can cope with sub-micron demands and large field of view applications. Certain key features are required to give a system the ability to perform well in both arenas.

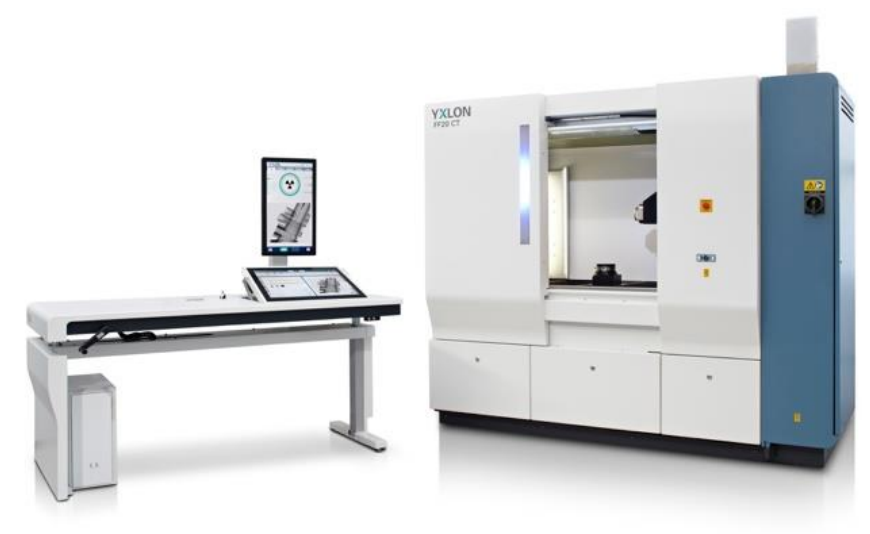

Figure 5 Cross Over System

A very stable base is needed for the key components (tube, manipulator and detector), normally Granite is used for this. Very accurate motion systems, with encoder modules made by Heidenheimer or other top quality manufacturers. Rotation tables being very high precision with high end air bearings, giving accurate and repeatable results. 


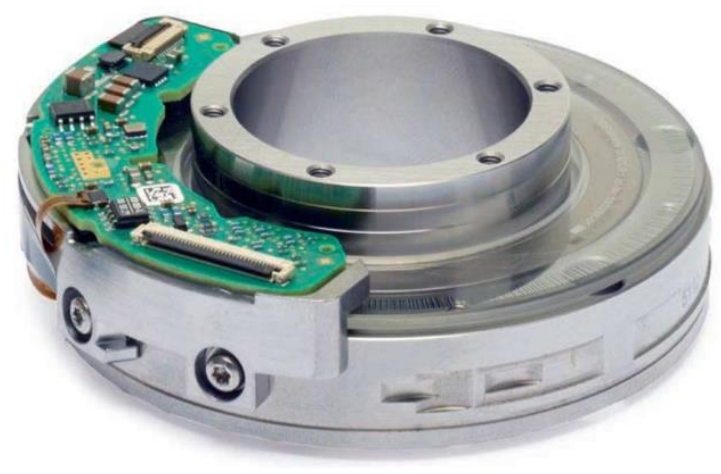

Figure 6 Air bearing rotation table

The higher-powered x-ray tubes also tend to be water cooled with diamond targets to give stable performance for demanding applications and over longer scanning times.

To have the ability to work at the Nano and sub-micron level and also to image larger demanding applications it is normal for these systems to have 2 tube types. This can add to the time needed, however some systems can change tubes at the touch of a button. Even keeping the same focus region when the tubes are changed, this is a great advantage

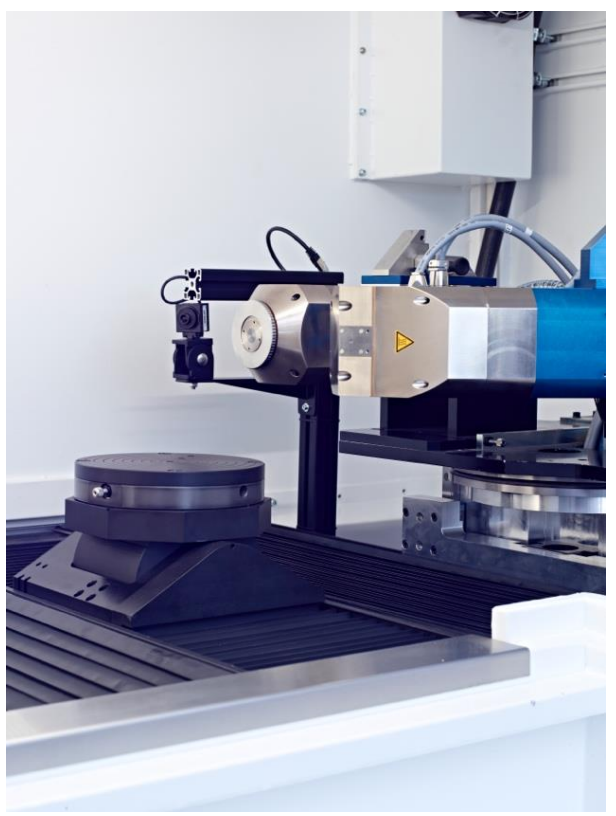

Figure 7 shows two $x$-ray tubes inside the system

These systems also tend to be larger than the normal off line ones seem in our industry. Partly due to the configuration which is horizontal rather than vertical, the larger and heavier sample sizes and the larger flat panel detectors.

\section{New Technologies}

\section{Helical scanning}

In order to increase the field of view but maintain high magnification helical scanning is used, as well as very accurate manipulation very clever software is needed to control the technology. The tube and the detector rise together allowing the field of view to move up the sample, producing very detailed large image.
The other alternative is to use stitching to join several smaller images together and produce one large image but the accuracy is poor by comparison.

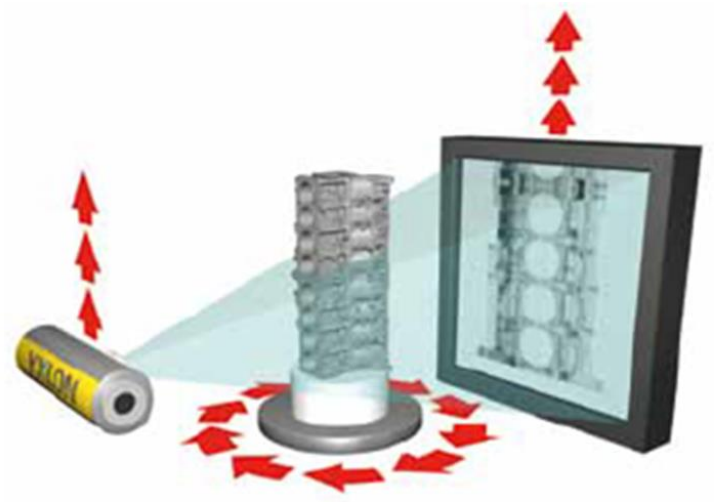

Figure 8 shows helical scanning layout

This allows very detailed images of larger objects to be made as the smart phone below illustrates

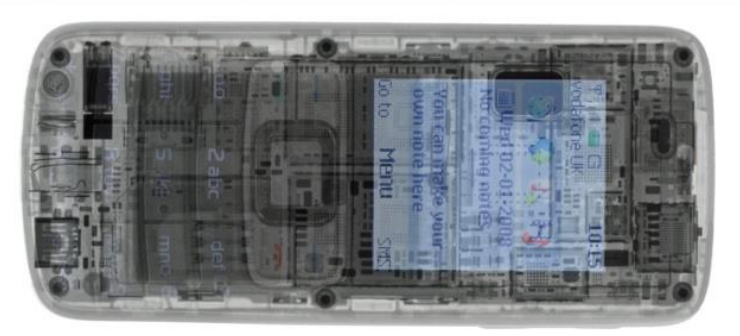

Figure 9 Smart phone imaged in a single shot

\section{Virtual Rotation Access}

This is a software driven technology which allows the operator to select a field of view not in the centre of the rotation table.

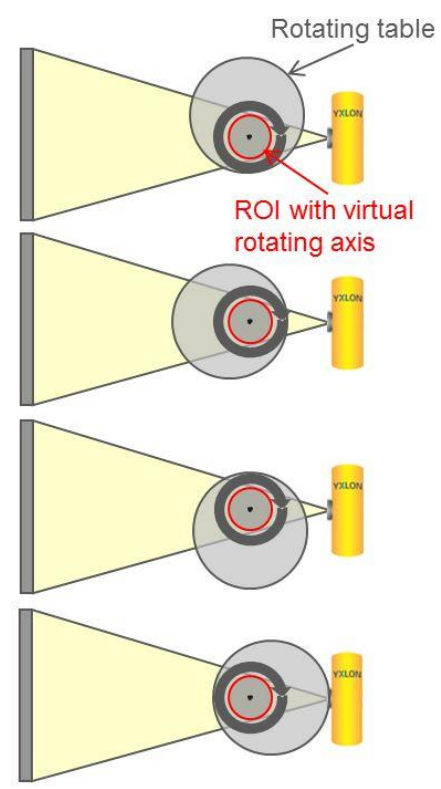

Figure 10 shows virtual rotation in action 
It's ideal for hard to hold samples or to make several 3D images without having to reposition the sample multiple times to make the optimum image

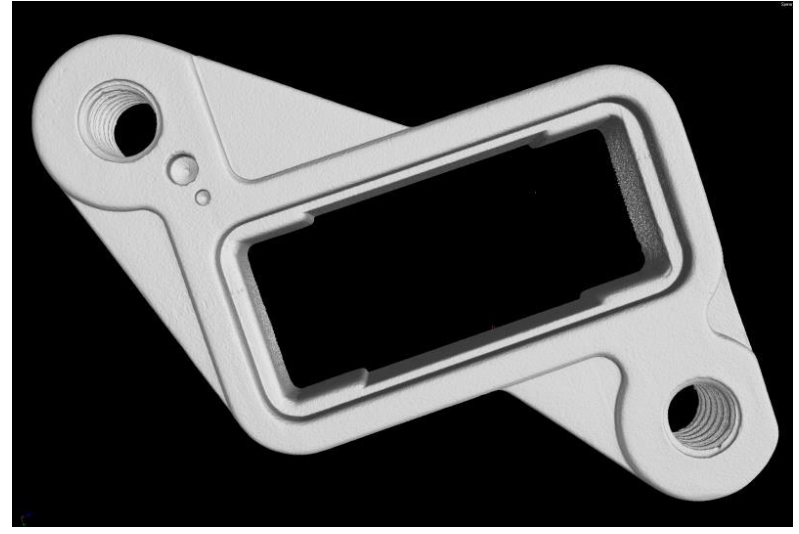

Figure 11a full 3D image of bracket

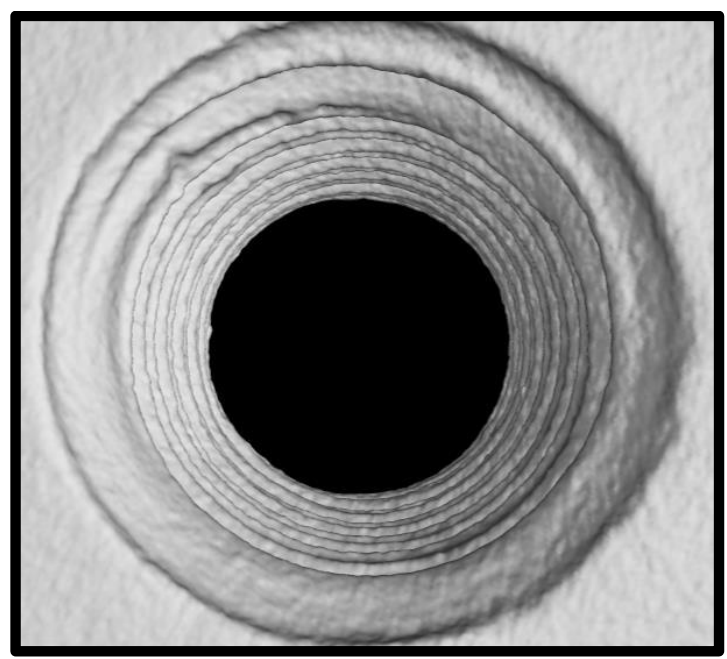

Figure 11b 3D image of mounting hole made by virtual rotation

\section{Anti-collision systems}

When working with large samples or with those which are offset or held in at an angle the potential for collision and damage increases. The more sophisticated systems use a camera and clever software to check the position of the sample during rotation. This allows the operator to set the sample close to the tube giving high magnification of the image without the potential for damage.

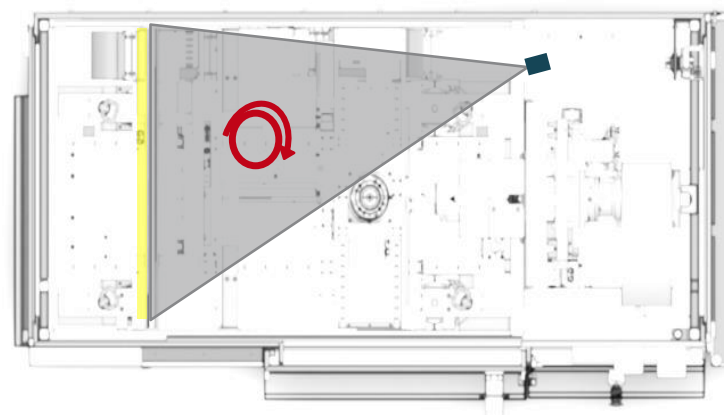

Figure 12 shows camera action for collision prevention

\section{Remote Monitoring and Push Messages}

This software innovation allows the operator to manage the system remotely or even manage multiple systems.

Continuous communication of inspection progress and health data gives the operator complete control. It displays the system Health Monitor, real time information and alerts. The software works on all Windows tablets and PCs; it allows remote monitoring sending push messages calling the user for direct system interaction e.g. decision expected or scan finished

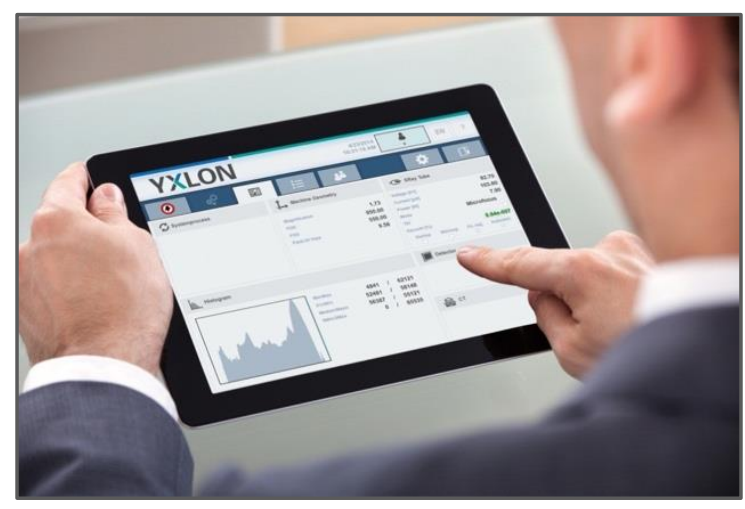

Figure 13 shows system remote monitor

\section{Metrology}

Traditional x-ray systems have struggled with measurement accuracy and repeatability, basically they were microscope based and not measurement systems.

Cross Over systems have much more accurate components and active vibration damping, making them metrology based.

The better machines have calibration values related to the PTB certified measurement sphere gauge in a controlled environment at $20^{\circ} \mathrm{C}+/-0.5^{\circ}$. Some companies offer guaranteed PTB values for a delivered system which can be ordered as optional feature.

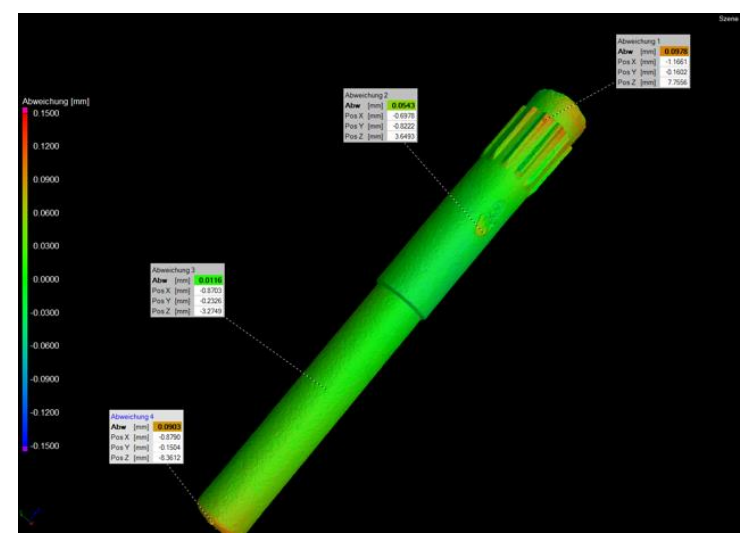

Figure 14 actual to nominal comparison measurement of component

\section{CT Images from High End Systems}

Below are some images demonstrating the capability of Cross Over systems, from sub-micron to full assemblies. They show features which are very challenging such as dendrites a few microns in diameter and hard to see even with high end 2D 
systems. Through $17 \mu \mathrm{m}$ diameter copper wires to batteries and relays up to smart phones

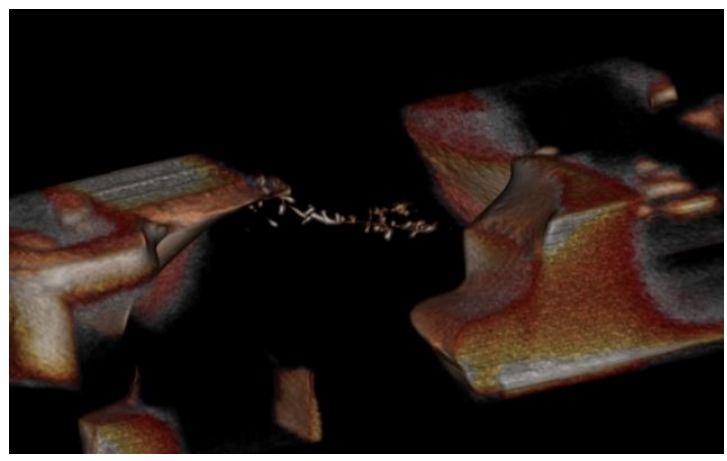

Figure 15 dendrites $<5 \mu \mathrm{m}$

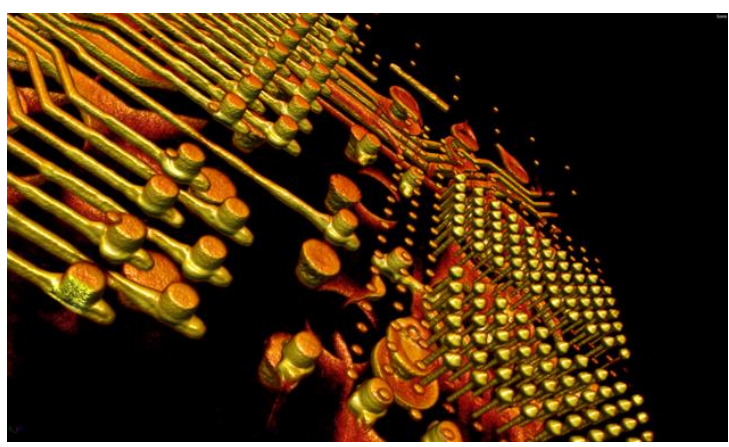

Figure 16 copper pillars

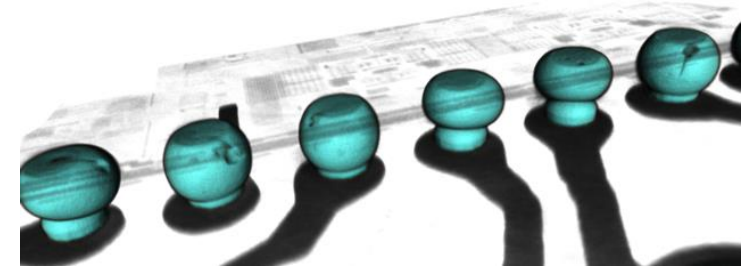

Figure $1730 \mu \mathrm{m}$ balls

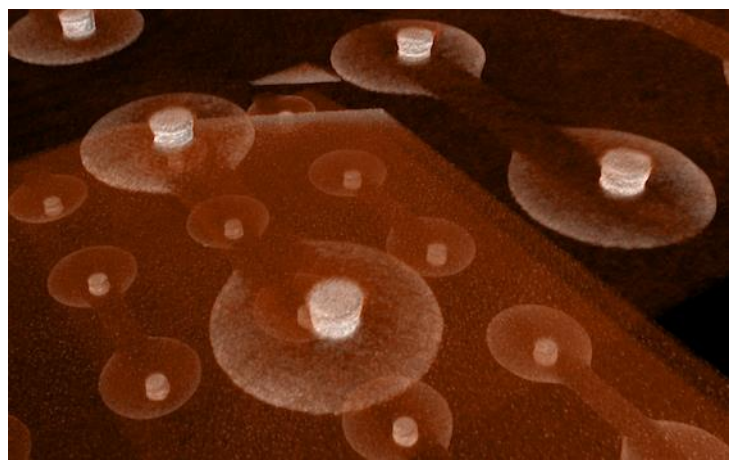

Figure $1812 \mu \mathrm{m}$ copper pillars

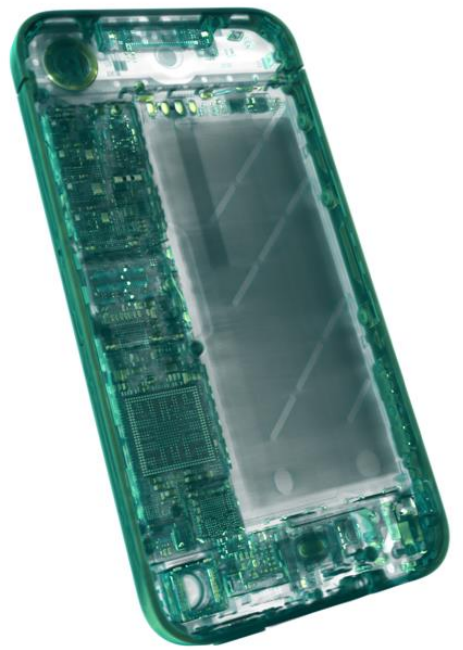

Figure 19 SMART phone, complete image

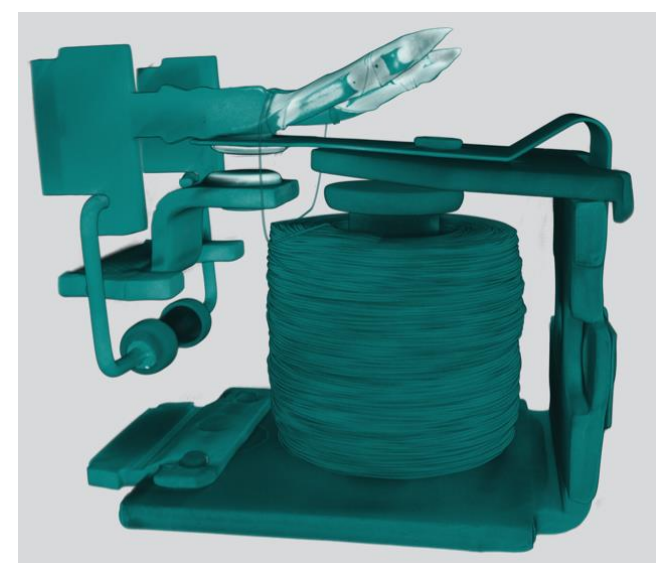

Figure 20 Relay assembly

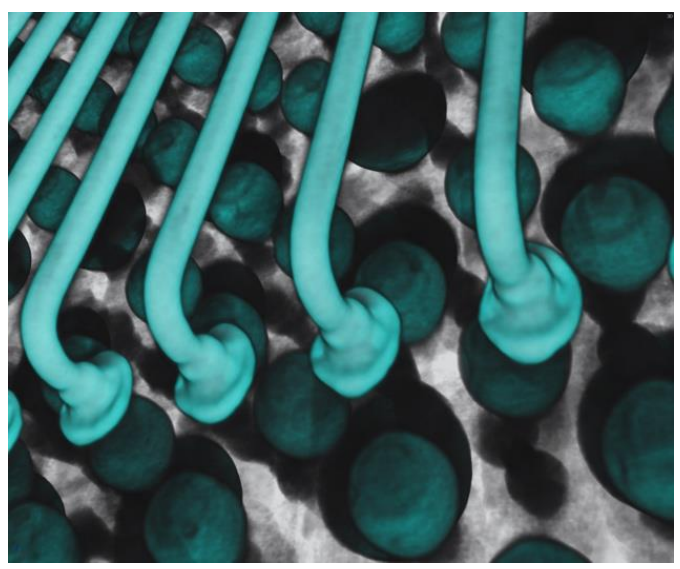

Figure $2115 \mu \mathrm{m}$ copper wires

\section{Acknowledgements}

Rene Sommer and Thorsten Rother of Yxlon International, for many of the CT images. Dr David Bernard, for his support on this paper. 